\title{
The evaluated nuclear data library for ADS in China
}

\author{
Yinlu Han ${ }^{1, \text { a }}$, Hairui Guo ${ }^{2}$, Yongli Xu ${ }^{3}$, Zhengjun Zhang ${ }^{4}$, Haiying Liang ${ }^{1}$, Zhendong $\mathrm{Wu}^{1}$, Xinwu $\mathrm{Su}^{3}$, Yue Zhang ${ }^{1}$, \\ Chonghai $\mathrm{Cai}^{5}$, and Qingbiao Shen ${ }^{1}$ \\ 1 Key Laboratory of Nuclear Data, China Institute of Atomic Energy, PO Box 275(41), Beijing 102413, P.R. China \\ 2 Institute of Applied Physics and Computational Mathematics, Beijing 100094, P.R. China \\ 3 School of Physics and Electronic Science, Shanxi Datong University, Datong 037009, P.R. China \\ ${ }^{4}$ Department of Physics, Northwest University, Xi' an 710069, P.R. China \\ 5 Department of Physics, Nankai University, Tianjin 300071, P.R. China
}

\begin{abstract}
The neutron and proton reaction data up to $200 \mathrm{MeV}$ are calculated and evaluated using both nuclear model calculations and experimental data for Accelerator Driven System (ADS). The evaluated methods and results of neutron and proton reaction data and present status of evaluated nuclear data library in China are introduced.
\end{abstract}

\section{Introduction}

The Accelerator-Driven System (ADS) is defined as a system driven by energetic particles of $\mathrm{GeV}$ range accelerated by a so-called high power accelerator incorporated normally with a target of a heavy element, which generates intense secondary particles, e.g., neutron, proton, etc., via the spallation nuclear process. The application of the scheme involves the nuclear transmutation for high level nuclear waste (HLW), plutonium burner for proliferation resistance, the energy production, fissile or tritium fuel breeding, neutron sources for material irradiation, neutron scattering science, industrial applications, and so on.

To address these needs, a program is under way to develop new evaluated nuclear data libraries for incident protons and neutrons up to $200 \mathrm{MeV}$ for a range of high-priority elements in the ENDF format. They require accurately nuclear reaction data of common cross sections and especially need the data of neutron and proton induced energy-angle correlated spectra of secondary light particles (neutron, proton, deuteron, triton, helium and alpha-particle) as well as double differential cross sections, $\gamma$-ray production cross sections and $\gamma$-ray production energy spectra. These evaluations are based on a combination of nuclear theoretical model calculations and measured data to evaluate cross sections.

This paper describes methods and results of the neutron and proton cross section evaluations and present status of evaluated nuclear data library in China.

\section{Theoretical models and parameters}

The optical model is used to describe measured neutroninduced total, nonelastic, elastic cross section and elastic scattering angular distributions, and the measured charged particles-induced total reaction cross sections and elastic scattering angular distributions, and to calculate the transmission coefficient of the compound nucleus and

a e-mail: hanyl@ciae.ac.cn the pre-equilibrium emission process for all particles (neutron, proton, deuteron, triton, helium and alphaparticle). The optical model potentials considered here are Woods-Saxon [1] type for the real part, Woods-Saxon and derivative Woods-Saxon type for the imaginary parts corresponding to the volume and surface absorptions and the Thomas form for the spin-orbit part, respectively. The theoretical model code APMN [2] is used to obtain optical model potential parameters. The best neutron and proton optical potential parameters for different targets are obtained from the experimental data at incident proton energy up to $250 \mathrm{MeV}$, respectively. The optical potential parameters for deuteron, triton, helium and alpha-particle are taken from the global optical potential parameters [3-6] with incident energies from threshold up to $200 \mathrm{MeV}$, based on the experimental data of particle total reaction cross sections and elastic scattering angular distributions, respectively.

The unified Hauser-Feshbach and exciton model [7] are used to describe the nuclear reaction equilibrium and preequilibrium decay processes at incident neutron energies below $20 \mathrm{MeV}$. The Hauser-Feshbach model with width fluctuation correction describes the emissions from compound nucleus to the discrete levels and continuum states of the residual nuclei in equilibrium processes, while the preequilibrium process is described by the angular momentum and parity dependent exciton model. The emissions to the discrete level and continuum states in the multi-particle emissions for all opened channels are included. The secondary particle emissions are described by the multi-step Hauser-Feshbach model at incident neutron energies below $20 \mathrm{MeV}$. The preequilibrium statistical theory based on the exciton model, the evaporation models and the Hauser-Feshbach theory with width fluctuation correction, and the intranuclear cascade model are used to describe the nuclear reaction preequilibrium and equilibrium decay processes for incident neutron energies above $20 \mathrm{MeV}$. The improved Iwamoto-Harada model [8-11] is used to describe the 


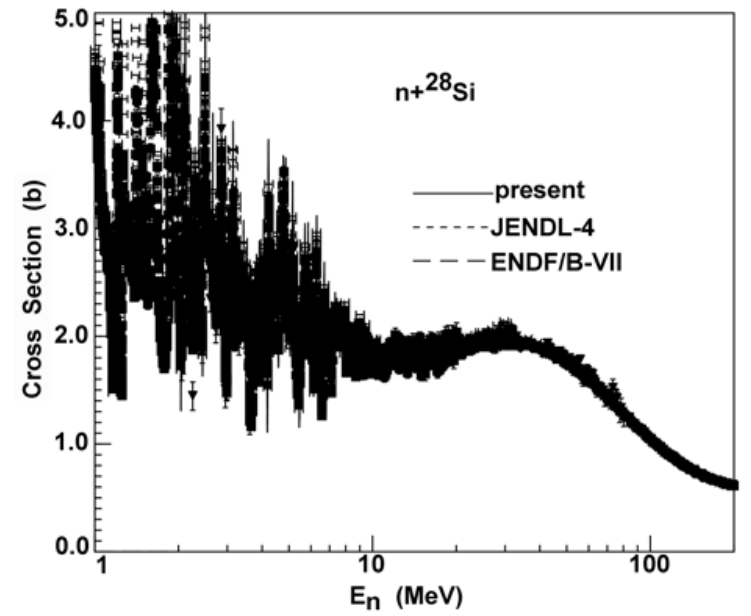

Figure 1. Calculated total cross sections of $n+{ }^{28} \mathrm{Si}$ reaction compared with the experimental data.

composite particle $\left(\mathrm{d}, \mathrm{t},{ }^{3} \mathrm{He}, \alpha\right)$ emission in compound nucleus. The improved Iwamoto-Harada model is included in the exciton model for the light composite particle emissions. Fission is included as a decay channel, that is, a fission competitive width can be estimated at every step of the cascades. The effective single-peak fission potential barrier is used to describe fission channels. The BohrWheeler theory $[12,13]$ is used in transmission coefficients computed at each barrier. The preequilibrium mechanism of $\gamma$-ray emission is also taken into account. The level density parameters and pair correction parameters of the Gilbert-Cameron level density [14] for low energy are used. The Ignatyuk model [15] is particularly appropriate for the relatively high energies and used. The direct reaction are calculated by the distorted wave Born approximation theory. The code DWUCK4 [14] of the distorted wave Born approximation theory is used to precalculate the direct inelastic scattering cross sections and angular distributions of discrete levels. The experimental data of inelastic scattering angular distributions for discrete levels are used to guide theoretical calculations. The deformation parameters for excited levels are obtained.

The UNF [17] code is used at incident neutron energies below $20 \mathrm{MeV}$. The MEND [18] code is used up to $200 \mathrm{MeV}$. The angular momentum and parity dependent exciton model is used in UNF code, other models are the same as MEND code. The parameters of the level densities and pair correction are taken from RIPL [19], and adjusted by fitting the experimental data of some channel reaction cross sections. Double differential cross sections can be calculated by generalized master equation to get the angular momentum dependent lifetime with the Legendre expansion form. In order to simplify the calculations, the angular dependent formula form of Kalbach phenomenological approach [20] is used in present calculation of the double differential cross sections for neutron, proton, deuteron, triton, helium and alphaparticle emissions above incident nucleon energies of $20 \mathrm{MeV}$.

\section{Theoretical results and analysis}

All cross sections of neutron induced reactions, angular distributions, energy spectra and double

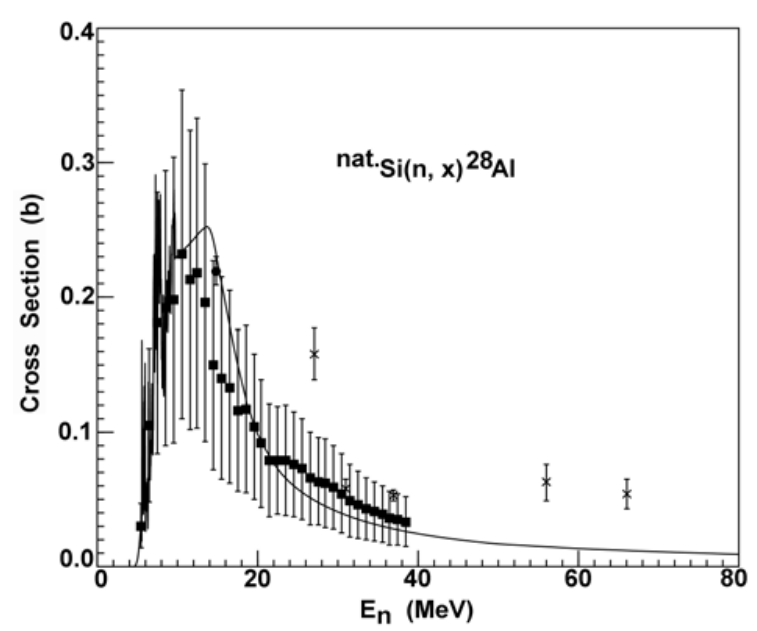

Figure 2. Calculated ${ }^{\text {nat. }} \mathrm{Si}(\mathrm{n}, \mathrm{x})^{28} \mathrm{Al}$ reaction cross sections compared with the experimental data.

differential cross sections are consistently calculated and evaluated for ${ }^{23} \mathrm{Na},{ }^{24,25,26} \mathrm{Mg},{ }^{27} \mathrm{Al},{ }^{28,29,30} \mathrm{Si},{ }^{31} \mathrm{P}$, ${ }^{32,33,34,36} \mathrm{~S},{ }^{40,42,43,44,46,48} \mathrm{Ca},{ }^{51} \mathrm{~V},{ }^{50,52,53,54} \mathrm{Cr},{ }^{54,56,57,58} \mathrm{Fe}$, ${ }^{59} \mathrm{Co}, \quad{ }^{58,60,61,62,64} \mathrm{Ni}, \quad{ }^{63,65} \mathrm{Cu}, \quad{ }^{90,91,92,94,96} \mathrm{Zr}, \quad{ }^{93} \mathrm{Nb}$, ${ }_{92,94,95,96,97,98,100 \mathrm{Mo},} \quad{ }_{107,109} \mathrm{Ag}, \quad{ }_{113,115} \mathrm{In}$, $152,154,155,156,157,158,160 \mathrm{Gd},{ }^{181} \mathrm{Ta},{ }^{180,182,183,184,186} \mathrm{~W},{ }^{197} \mathrm{Au}$, 204,206,207,208 $\mathrm{Pb},{ }^{209} \mathrm{Bi},{ }^{232} \mathrm{Th},{ }^{237} \mathrm{~Np},{ }^{232-240} \mathrm{U},{ }^{236,244-246} \mathrm{Pu}$, $241,242 \mathrm{~m}, 242,243 \mathrm{Am}$ and ${ }^{243-248} \mathrm{Cm}$ at incident neutron energies below $200 \mathrm{MeV}$, based on the nuclear theoretical models and experimental data.

All cross sections of proton induced reactions, angular distributions, energy spectra and double differential cross sections are consistently calculated and evaluated for ${ }^{27} \mathrm{Al},{ }^{28,29,30} \mathrm{Si},{ }^{40,42,43,44,46,48} \mathrm{Ca},{ }^{50,52,53,54} \mathrm{Cr}$, ${ }^{54,56,57,58} \mathrm{Fe},{ }^{59} \mathrm{Co},{ }^{58,60,61,62,64} \mathrm{Ni},{ }^{63,65} \mathrm{Cu},{ }^{90,91,92,94,96} \mathrm{Zr}$, 92,94,95,96,97,98, ${ }_{100} \mathrm{Mo}, \quad{ }^{181} \mathrm{Ta}, \quad{ }^{180}, 182,183,184,186 \mathrm{~W}, \quad{ }^{197} \mathrm{Au}$, $204,206,207,208 \mathrm{~Pb},{ }^{209} \mathrm{Bi},{ }^{232} \mathrm{Th},{ }^{235,238} \mathrm{U}$ and ${ }^{239} \mathrm{Pu}$ at incident proton energies below $200 \mathrm{MeV}$.

Theoretical calculated results are compared with existing experimental data, and the evaluated results from ENDF/B-VII [21] and JENDL-4 [22]. Good agreement is generally observed between the calculated results and the experimental data. The theoretical models provide the good description of the shapes and magnitude of the energy spectra and double differential cross section of emission neutron, proton, deuteron, triton, helium and alpha. The evaluated and calculated data is stored using ENDF/B high-energy format.

The comparisons of some calculated and evaluated results for $n+{ }^{28,29,30 \text {,nat. }}$ Si reactions with the experimental data are only given in this paper. The experimental data of all cross sections are taken from EXFOR Library. The calculated and evaluated results of total cross sections for $\mathrm{n}+{ }^{28} \mathrm{Si}$ reaction are in good agreement with the experimental data [23] of $n+{ }^{\text {nat. }}$ Si reaction as shown in Fig. 1. The calculated results for ${ }^{\text {nat. }} \mathrm{Si}(\mathrm{n}, \mathrm{x})^{28} \mathrm{Al}$ reaction are compared with the experimental data as shown in Fig. 2. The calculated results from ${ }^{28} \mathrm{Si}(\mathrm{n}, \mathrm{p}){ }^{28} \mathrm{Al},{ }^{29} \mathrm{Si}(\mathrm{n}$, $\mathrm{np}+\mathrm{d})^{28} \mathrm{Al}$ and ${ }^{30} \mathrm{Si}(\mathrm{n}, \mathrm{nd}+\mathrm{p} 2 \mathrm{n}+\mathrm{t})^{28} \mathrm{Al}$ reactions are in agreement with the experimental data [24].

Based on the agreements of the calculated results with the experimental data for all reaction cross sections and angular distributions, the energy spectra as well as double differential cross sections for neutron, proton, deuteron, 

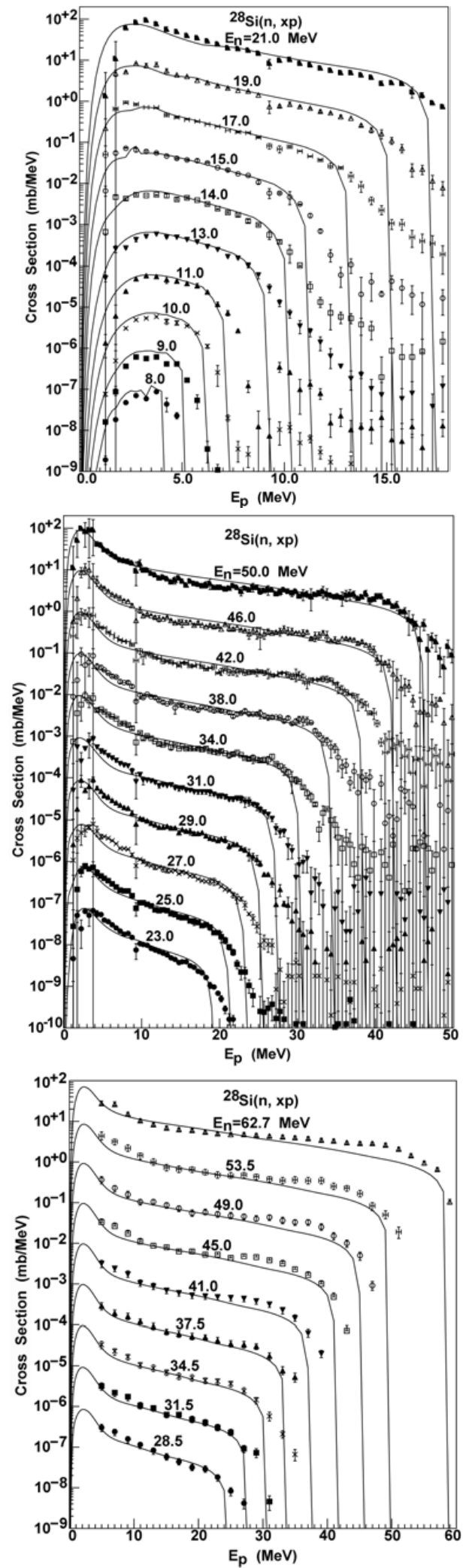

Figure 3. Calculated proton energy spectra compared with the experimental data for $\mathrm{n}+{ }^{28} \mathrm{Si}$ reactions.

triton, helium and alpha emission, $\gamma$-ray production cross sections and $\gamma$-ray production energy spectrum are calculated by the theoretical models. The calculated results of proton emission energy spectra for $\mathrm{n}+{ }^{28} \mathrm{Si}$ reaction are compared with the experimental data $[25,26]$ at incident neutron energies 8.0 to $62.7 \mathrm{MeV}$ as shown in Fig. 3. The
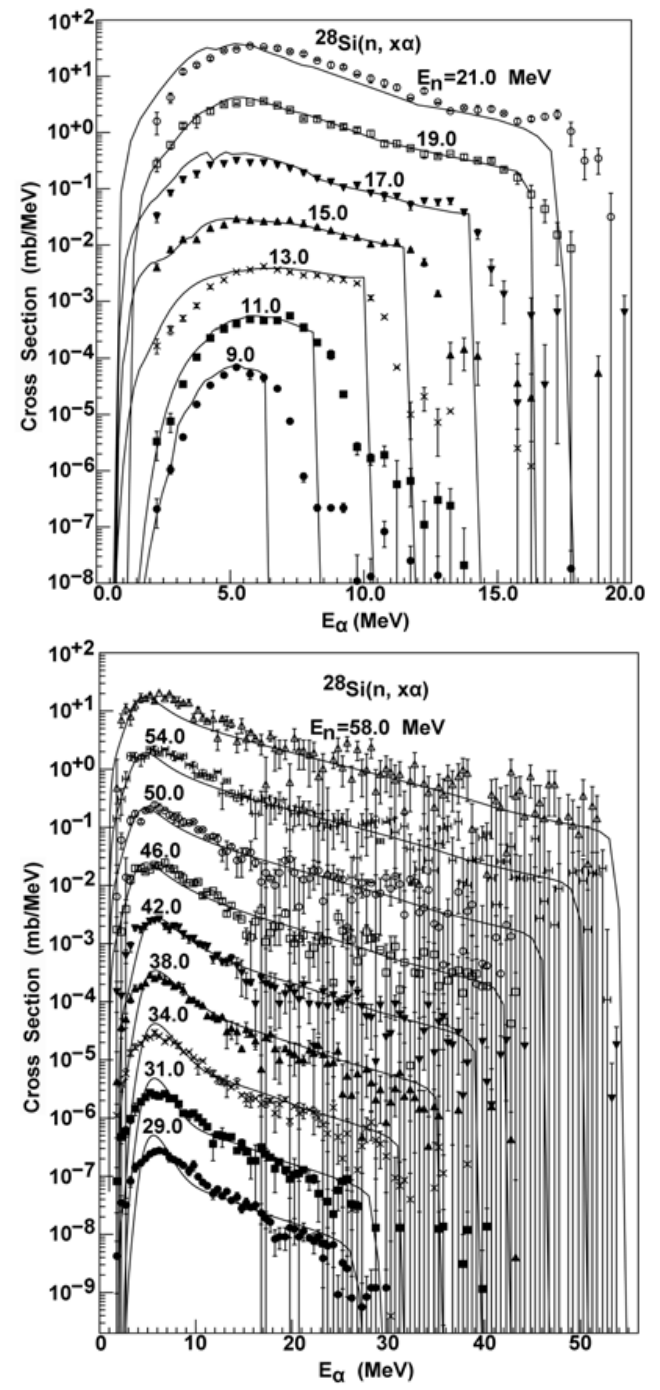

Figure 4. Calculated alpha energy spectra compared with the experimental data for $\mathrm{n}+{ }^{28} \mathrm{Si}$ reactions.

shape and magnitude of the calculated results curve are in good agreement with those of the experimental data.

The calculated results for alpha emission energy spectra are compared with the experimental data [25] at incident neutron energies 9.0 to $58.0 \mathrm{MeV}$ as shown in Fig. 4. The shape and magnitude of the calculated results curve for alpha emission are in good agreement with those of the experimental data in all alpha particles emission energy. The general trend observed is a decreasing emission probability with increasing emission alpha energy. A quasi-isotropic component is observed at very low energy $(0-8 \mathrm{MeV})$, where are mainly from the contributions of equilibrium reaction.

The calculated results of the double differential cross sections of deuteron, triton and helium emission for $n+{ }^{28} \mathrm{Si}$ reaction are compared with the experimental data [25-27]. The shape and magnitude of the calculated results curve is in good agreement with those of the experimental data as shown in Figs. 5 to 7.

This work was part of National Basic Research Program of China (973 Program), and was supported by the China Ministry of Science and Technology under Contract No. 2007CB209903. 


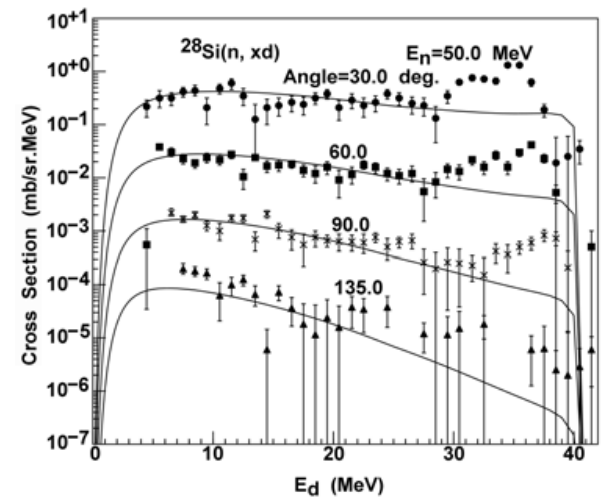

Figure 5. Calculated double differential cross sections of deuteron emission compared with the experimental data for $\mathrm{n}+{ }^{28} \mathrm{Si}$ reaction.
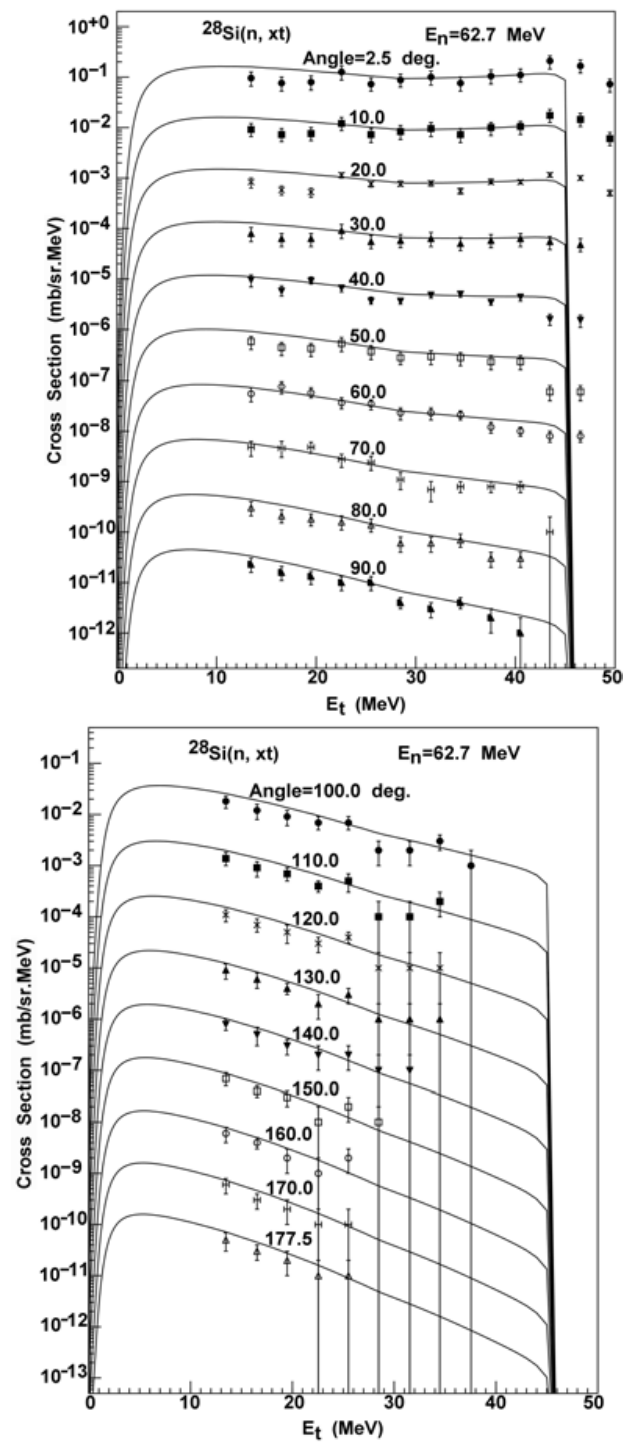

Figure 6. Calculated double differential cross sections of triton emission compared with the experimental data for $n+{ }^{28} \mathrm{Si}$ reaction.

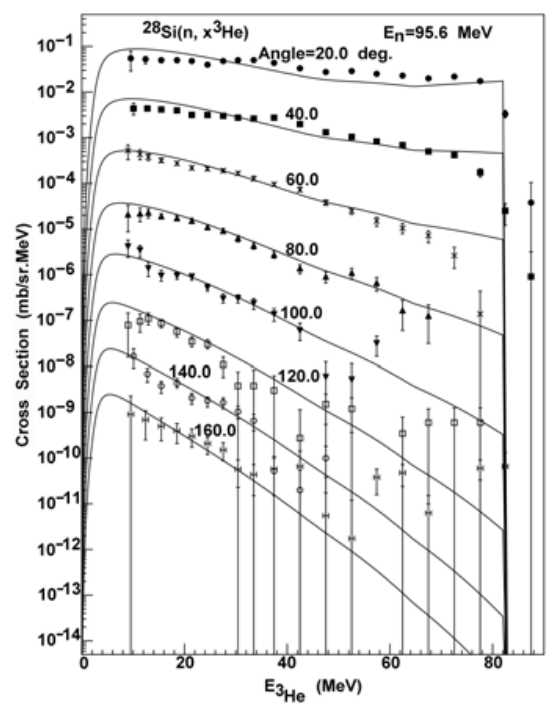

Figure 7. Calculated double differential cross sections of helium emission compared with the experimental data for $n+{ }^{28} \mathrm{Si}$ reaction.

\section{References}

[1] JR. F. D. Becchetti, G.W. Greenlees, Phys. Rev. 182, 1190 (1969)

[2] Qingbiao Shen, Nucl. Sci. Eng. 141, 78 (2002)

[3] Yinlu Han, et al., Phys. Rev. C 74, 044615 (2006)

[4] Yongli Xu, Hairui Guo, Yinlu Han, Qingbiao Shen, Sci. China Phys. Mech. Astron. 54, 2005 (2011)

[5] Yongli Xu, Hairui Guo, Yinlu Han, Qingbiao Shen, Int. J. Mod. Phys. E 24, 1550005 (2015)

[6] Xinwu Su, Yinlu Han, Int. J. Mod. Phys. E 24, 1550092 (2015)

[7] Jingshang Zhang, Nucl. Sci. Eng. 114, 55 (1993)

[8] A. Iwamoto, K. Harada, Phys. Rev. C 26, 1821 (1982)

[9] J.S. Zhang, et al., Z. Phys. A 344, 251 (1992)

[10] J.S. Zhang, Nucl. Sci. Eng. 116, 35 (1994)

[11] Qingbiao Shen, Nucl. Sci. Eng. 117, 99 (1994)

[12] N. Bohr, J.A. Wheeler, Phys. Rev. 56, 426 (1939)

[13] D.L. Hill, J.A. Wheeler, Phys. Rev. 89, 1102 (1953)

[14] W. Dilg, et al., Nucl. Phys. A 217, 269 (1973)

[15] A.V. Ignatyuk, et al., Sov. J. Nucl. Phys. 21, 255 (1975)

[16] P.D. Kunz, Distorted Wave Code DWUCK4, University of Colorado (1994)

[17] Jingshang Zhang, Nucl. Sci. Eng. 142, 207 (2002)

[18] Chong-hai Cai, Nucl. Sci. Eng. 153, 93 (2006)

[19] R. Capote, et al., Nucl. Data Sheets 110, 3107 (2009)

[20] C. Kalbach, Z. Phys. A 283, 401 (1977)

[21] M.B. Chadwick et al., Nucl. Data Sheets 107, 2931 (2006)

[22] K. Shibata et al., J. Nucl. Sci. Technol. 48, 1 (2011)

[23] R.W. Finlay, et al., Phys. Rev. C 47, 237 (1993)

[24] Y. Uwamino, et al., Nucl. Sci. Tech. 31, 1 (1994)

[25] F.B. Bateman, et al., Phys. Rev. C 60, 064609 (1999)

[26] S. Benck, et al., Nucl. Sci. Eng. 141, 55 (2002)

[27] U. Tippawan, et al., Phys. Rev. C 69, 064609 (2004) 